\title{
A Novel Spike-Wave Discharge Detection Framework Based on the Morphological Characteristics of Brain Electrical Activity Phase Space in an Animal Model
}

\author{
Saleh Lashkari $^{\circledR}$, Ali Moghimi ${ }^{\circledR}$, Hamid Reza Kobravi ${ }^{{ }^{\circledR}}$, Mohamad Amin Younessi Heravi ${ }^{*}{ }^{(\mathbb{D}}$ \\ ${ }^{1}$ Department of Biomedical Engineering, Imam Reza International University, Mashhad, Iran \\ ${ }^{2}$ Rayan Center for Neuroscience \& Behavior, Department of Biology, Faculty of Science, Ferdowsi University of Mashhad, \\ Mashhad, Iran \\ ${ }^{3}$ Biomedical Engineering Research Center, Mashhad Branch, Islamic Azad University, Mashhad, Iran \\ ${ }^{4}$ Department of Radiology and Medical Physics North Khorasan University of Medical Sciences, Bojnurd, Iran
}

\begin{abstract}
Background: Animal models of absence epilepsy are widely used in childhood absence epilepsy studies. Absence seizures appear in the brain's electrical activity as a specific spike wave discharge (SWD) pattern. Reviewing long-term brain electrical activity is time-consuming and automatic methods are necessary. On the other hand, nonlinear techniques such as phase space are effective in brain electrical activity analysis. In this study, we present a novel SWD-detection framework based on the geometrical characteristics of the phase space.

Methods: The method consists of the following steps: (1) Rat stereotaxic surgery and cortical electrode implantation, (2) Long-term brain electrical activity recording, (3) Phase space reconstruction, (4) Extracting geometrical features such as volume, occupied space, and curvature of brain signal trajectories, and (5) Detecting SDWs based on the thresholding method. We evaluated the approach with the accuracy of the SWDs detection method.

Results: It has been demonstrated that the features change significantly in transition from a normal state to epileptic seizures. The proposed approach detected SWDs with 98\% accuracy.

Conclusion: The result supports that nonlinear approaches can identify the dynamics of brain electrical activity signals.

Keyword: Absence epilepsy; EEG, WAG/Rij; Animal model; Phase space; Geometrical features.
\end{abstract}

*Correspondence to Mohamad Amin Younessi Heravi, Department of Radiology and Medical Physics, Medicine School, Shahriar St, Bojnurd North Khorasan, Bojnurd, Iran. Tel: +98-5842296787, Email: a.younessi7@ gmail.com

Published online 30 October 2021

Citation: Lashkari S, Moghimi A, Kobravi HR, Younessi Heravi MA. A novel spike-wave discharge detection framework based on the morphological characteristics of brain electrical activity phase space in an animal model. Clin Neurosci J. 2021;8(4):180-187. doi:10.34172/icnj.2021.36.

\section{Introduction}

Epilepsy is one of the most common neurological disorders, afflicting $1 \%$ of the population. Currently, patients with epilepsy suffer from social problems and momentary lapses of consciousness. Absence seizures are general seizures, which highly occur in children between 4-12 years old and adolescents with juvenile absence epilepsy. The absence seizures appear in electroencephalogram (EEG) signals with particular spike-wave discharges (SWDs) with a frequency of about $3 \mathrm{~Hz}$ and a short duration (5-30 seconds). ${ }^{1,2}$

Wistar Albino Glaxo of Rijswijk (WAG/Rij) and genetic absence epilepsy rats from Strasbourg (GAERS) are wellaccepted experimental models of absence epilepsy. ${ }^{1,2}$ Because of the similar pharmacological and electrical characteristics, these models are extensively useful for human absence epilepsy. ${ }^{3}$

The EEG quantification is essential for three reasons. First, it is crucial to investigate the development of an antiepileptic drug. The dose of the drugs is directly related to the number of SWDs. So, SWDs detection can help assess drug efficacy. ${ }^{4}$ Secondly, new therapeutic methods such as surgical and deep brain stimulation (DBS) are more popular recently. ${ }^{5}$ The SWDs detection is the basis of these methods. If we identify the pre-seizure state, the appropriate stimulation pattern is applied at the right time. Thirdly, doctors require to score SWDs in longterm EEG. This task is performed manually ${ }^{6}$ and is prone to error. To this end, automatic SWDs scoring would be highly appreciated, since it helps fast quantification and

(C) 2021 The Author(s). This is an open access article distributed under the terms of the Creative Commons Attribution License (http:// creativecommons.org/licenses/by/4.0/), which permits unrestricted use, distribution, and reproduction in any medium, provided the original work is properly cited. 
facilitates clinical application techniques. ${ }^{7}$

There are very few studies in the literature related to automated SWD detection in animal models. Andrade et $\mathrm{al}^{8}$ proposed an offline SWDs detection framework based on the time-frequency analysis in the genetic model of absence epilepsy. The SWDs were detected using some features in certain frequency bands. Spontaneous seizures can be detected in the post-traumatic rat model of epilepsy. ${ }^{9}$ In this study, continuous long-term video EEGs were recorded to detect epileptic activity. The proposed method searched power peaks and then marked the onset of seizures. Sitnikova and colleagues ${ }^{10}$ used fast Fourier parameters and continuous Morlet wavelet transform to discriminate SWDs from sleep spindles in EEG signals from WAG/Rij model of epilepsy. They showed that EEG patterns of SWDs have different time-frequency parameters compared with sleep spindles. In Fanselow et al study, ${ }^{11}$ an automatic SWDs detection framework was developed based on EEG signals derivate. The SWDs were detected when the signals value derivate exceeds the threshold value. Another study ${ }^{12}$ introduced a method based on the amplitude values of EEG signals and the thresholding method. The method detected SWDs when EEG amplitude exceeded from absolute amplitude threshold. However, these studies are not based on the complex nature of the brain signal and cannot be used to identify the chaotic behaviors of the brain signal. According to the chaos and nonlinear dynamic theory, biomedical signals have complex behavior, and these methods are suitable for modeling them. There are several feature extraction techniques to identify the dynamic of chaotic signals. Fractal dimension, ${ }^{13,14}$ correlation dimension, ${ }^{15-19}$ entropy, ${ }^{20-24}$ Lyapunov exponent, ${ }^{25-28}$ and recurrence quantification analysis are common methods to analyze EEG signals.

However, these methods quantify the global characteristics of phase space and cannot identify the details of phase space. So, introducing the features which can detect the details of phase space are more effective. We proposed a novel method to detect SWDs based on the two-dimensional phase space's geometric features. ${ }^{29}$ We showed that our proposed method had more outstanding results than previously published approaches. Application of the method in an animal research setting, however, has revealed some aspects that required further improvement. In particular, the method only introduced statistical features from two-dimensional phase space while EEG system has higher-order dimensions, and this reduction may lead to erroneous results. In addition, trajectory changes have dynamic behaviors, and we have to consider this issue in our method. In this study, we tackle this problem by presenting a novel method to track the changes in phase space trajectory. ${ }^{30,31}$ We assumed that the SWDs have geometry in phase space as well as in the time domain and geometrical characteristics are helpful in predicting and detecting absence seizures. Among the novelties are: (1) Introducing morphological features in high dimensional phase space from EEG signals, (2) Improving the detection method by modifying thresholding method using a dynamical threshold, and (3) Using a benchmark oscillator-like Lorentz system to investigate the robustness of the proposed features. First, we introduce the subjects and procedure of surgery and electrode implantation. In the second step, we present the phase space reconstruction and feature extraction methods. Finally, we discuss our results and compare the performance of the proposed method with existing literature.

\section{Materials and Methods \\ Ethical Consideration}

This experimental study was done in the animal laboratory of Ferdowsi University, Mashhad, Iran. It was approved by the natural ethics committee (IR.NKUMS.REC.00.036) The animals were housed in pairs with free access to water and food. After surgery, they were housed individually. All procedures were done in accordance with the national guidelines for animal care and handling. Efforts were made to minimize the pain/distress experienced by the animals. Also, the least possible number of animals necessary to produce reliable data was used.

\section{Subjects and surgery}

All experiments were performed on 15 male WAG/Rij rats with at least 13 weeks of age (weight $300 \pm 5 \mathrm{~g}$ ). The rats were anesthetized with xylazine $11 \mathrm{mg} / \mathrm{kg}$ and ketamine $110 \mathrm{mg} / \mathrm{kg}$. Next, the skull was fixed with a stereotax, and cotton and alcohol were used to prepare the surface of the skull. In addition, phenylephrine was used to prevent bleeding. In all animals, a twisted Bipolar electrode was implanted on the frontal cortex $(1 \mathrm{~mm}$ above Bregma and $3 \mathrm{~mm}$ from Lambda) and the reference electrode was implanted on the parietal cortex. An extra screw was used to fix dental cement. All coordinates were determined according to the stereotaxic atlas of Paxinos and Watson. The rats were allowed recovery for a week.

\section{Brain Electrical Activity Recording}

After recovery, each animal was kept in the individual brain electrical activity record cage. Next, the brain electrical activity electrode was connected to the recording system. The signals were recorded by $1 \mathrm{kHz}$ sampling rate and amplified with PowerLab (AD Instrumentation Co, Australia). The recording was performed continuously for 24 to 74 hours. Figure 1 shows the coordination of the recording electrode, electrode implantation, and a sample brain electrical activity recorded.

\section{Phase Space Reconstruction}

The phase space is one of the effective methods in 


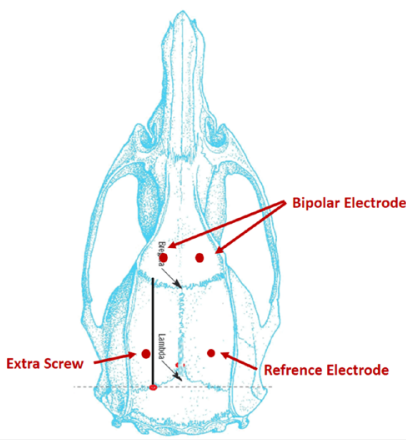

(a)

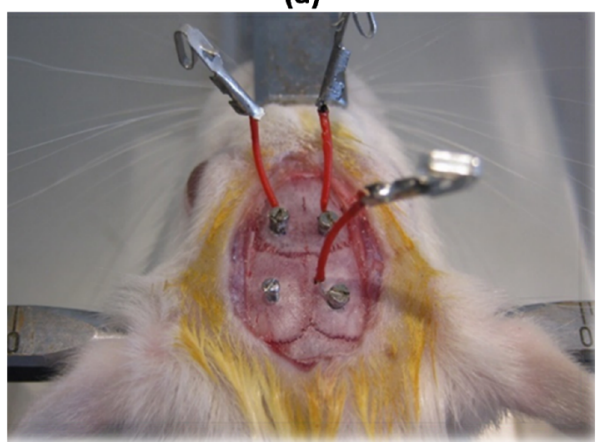

(b)

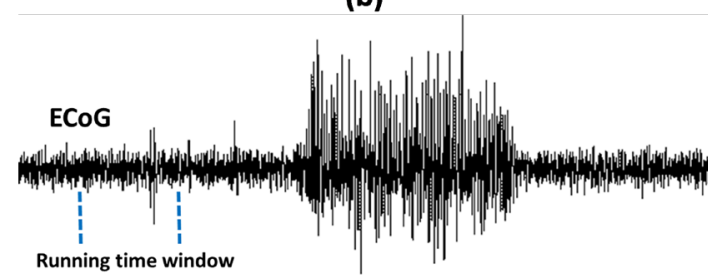

Figure 1. (a) The rat skull atlas and the coordinates of the recording electrode. (b) Bipolar, reference electrode, and extra screw implantation. (c) . A sample of recorded brain electrical activity includes SWDs with $3 \mathrm{~Hz}$.

biomedical signal processing. In the biological system, we can only identify the dynamics of the system with these observations because we do not have access to the system equations and only have observations of the system such as brain electrical activity. Taken theor $y^{32}$ explains that the phase space can be reconstructed with the time-delayed series of observations. According to this theory, if $\mathrm{x}(\mathrm{t})$ is a time series, the delayed phase-space vectors are estimated with the following equation:

$X(i)=\left[\begin{array}{l}x(i) \cdot x(i+\tau) \cdot x(i+2 \tau) \\ \ldots \ldots . x(i+(m-1) \tau)\end{array}\right]$

$\mathrm{i}=1.2 \ldots . . \mathrm{N}-(\mathrm{m}-1) \tau$

Where $\tau$ is a time delay, and $m$ is the embed dimension which can be calculated by the false nearest-neighbor ${ }^{33}$ and the mutual information ${ }^{34,35}$ algorithm, respectively.

\section{Feature Extraction}

In this part, we quantify the trajectory of the state variable based on the geometrical properties of phase space.

\section{Enclosed Volume (EV)}

The EV quantifies the surrounded space by the trajectory; we introduce features to demonstrate the geometrical characteristics of trajectory in the phase space.

\section{Centroid}

To calculate a centroid of a set of $\mathrm{K}$ points, the averages of their coordinates are calculated. The centroid in twodimension space was calculated using the following equations:

Where $\mathrm{K}$ is the number of points in the phase space and the coordination of each point and $C_{x}$ and $C_{y}$ are the centroid components in Cartesian coordination.

$$
\begin{aligned}
& C_{x}=\frac{1}{K} \sum_{i=1}^{K} x_{i} \\
& C_{y}=\frac{1}{K} \sum_{i=1}^{K} y_{i} \\
& C=\sqrt{C_{x}{ }^{2}+C_{y}{ }^{2}}
\end{aligned}
$$

\section{Center of Gravity}

Center of gravity is calculated as follows:

$G_{x}=\frac{1}{6 A} \sum_{i=1}^{n-1}\left(x_{i}+x_{i+1}\right)\left(x_{i} y_{i+1}-x_{i+1} y_{i}\right)$

$G_{y}=\frac{1}{6 A} \sum_{i=1}^{n-1}\left(y_{i}+y_{i+1}\right)\left(x_{i} y_{i+1}-x_{i+1} y_{i}\right)$

$G=\sqrt{G_{x}^{2}+G_{y}^{2}}$

Where $\mathrm{n}$ is the number of samples, $x_{i}$ and $y_{i}$ are the coordination of each point in the Cartesian coordination, $G_{x}$ and $G_{y}$ are the center of gravity components and $\mathrm{A}$ is the polygon signed area:

$A=\frac{1}{2} \sum_{i=1}^{n-1}\left(x_{i} y_{i+1}-x_{i+1} y_{i}\right)$

\section{Occupied Space}

Two quantifiers present occupied space according to the centroid and center of gravity:

$O C=\frac{1}{K} \sum_{k=1}^{K} \overrightarrow{\boldsymbol{x}}(k)-C$

and

$O G=\frac{1}{K} \sum_{k=1}^{K} \overrightarrow{\boldsymbol{x}}(k)-G$

Where $\overrightarrow{\boldsymbol{x}}(k)$ is the trajectory vector, $\mathrm{K}$ is the number of samples, $\mathrm{C}$ and $\mathrm{G}$ present centroid and center of gravity, respectively.

Two features characterize the variation of occupied space 
according to the distance of samples of $\overrightarrow{\boldsymbol{x}}(t)$ to the centroid $(\mathrm{C})$ and center of gravity $(\mathrm{G})$, respectively. These features are calculated as follows:

$$
\begin{aligned}
& O C V=\sqrt{\frac{1}{K} \sum_{k=1}^{K} \overrightarrow{\boldsymbol{x}}(k)-C-O C^{2}} \\
& O G V=\sqrt{\frac{1}{K} \sum_{k=1}^{K} \overrightarrow{\boldsymbol{x}}(k)-G-O G^{2}}
\end{aligned}
$$

Where $O C V$ and $O G V$ characterize the variation of the convex hull in the attractor according to the centroid and center of gravity.

\section{Curvature}

Curvature represents the complex behavior of the trajectory. Curvature vector is calculated by the following formula:

$$
K=\frac{2 \overrightarrow{v_{1}} \overrightarrow{v_{2}}}{\overrightarrow{v_{1}} \overrightarrow{v_{2}} \overrightarrow{v_{1}}+\overrightarrow{v_{2}}}
$$

$\vec{K}(\vec{x}(t))=\left(K\left(x_{i}\right) \cdot K\left(x_{i+1}\right)\right.$.

$K\left(x_{i+2}\right) \ldots \ldots . K\left(x_{i+N}\right)$

Where $\overrightarrow{v_{1}}$ and $\overrightarrow{v_{2}}$ are the vectors between each sample and reference point, and $\vec{K}(\vec{x}(t))$ is the curvature vector. The curvature can be quantified by two quantifiers:

$$
\begin{aligned}
A K & =\frac{1}{K-2} \sum_{k=2}^{K-1} \vec{K}(\overrightarrow{\boldsymbol{x}}(t)) \\
K V & =\sqrt{\frac{1}{K-2} \sum_{k=2}^{K-1} \vec{K}(\overrightarrow{\boldsymbol{x}}(t))-A K^{2}}
\end{aligned}
$$

Where AK presents the curvature average of each trajectory sample and KV quantifies the overall changes in a trajectory. The value of AK increases when the attractor tends to complex shape. The variation of curvature is increased when the state of EEG signal was changed from normal to epileptic state.

\section{Seizure Detection Algorithm}

For detection of SWDs, a thresholding method for the features was applied. We developed the algorithm by applying an adaptive thresholding method, i.e., the parameters of thresholding were dynamically tuned by estimating the corresponding average values of the precursing indicators in a time window.

\section{Data Analysis}

We evaluated our method according to the two aspects: First, we evaluated the features to investigate their abilities to introduce a chaotic quantifier. The appropriate chaotic quantifiers must have two features. First, the chaotic quantifiers should not be sensitive to the changes in the initial condition of the chaotic system. Second, the quantifier is changed significantly by the variation of the chaotic system parameters. In this study, the conditions were evaluated by the Lorenz system ${ }^{36}$ with the following equation:

$$
\text { Lorenz system }:\left\{\begin{array}{c}
\frac{d X}{d t}=\sigma(Y-Z) \\
\frac{d Y}{d t}=r X-Y-X Z \\
\frac{d Z}{d t}=X Y-\beta Z
\end{array}\right.
$$

Where $\mathrm{X}, \mathrm{Y}$, and $\mathrm{Z}$ are the state variables, and $\sigma, r, \beta$ are the parameters of the Lorenz system. To investigate the sensitivity of the initial point, we used $t$ test. The attractor geometry of a chaotic system is related to the changes in the trajectory behavior of the system. So, a geometrical feature is sensitive to the changes in the dynamic trajectory. An appropriate quantifier is invariant to the changes of the initial point. On the other hand, efficient quantifiers significantly change by changing the system parameters. Second, we assessed the performance of the SWD detection system by the accuracy and time prediction according to the different thresholds.

Result

Figure 2 shows the boxplot of each feature for different
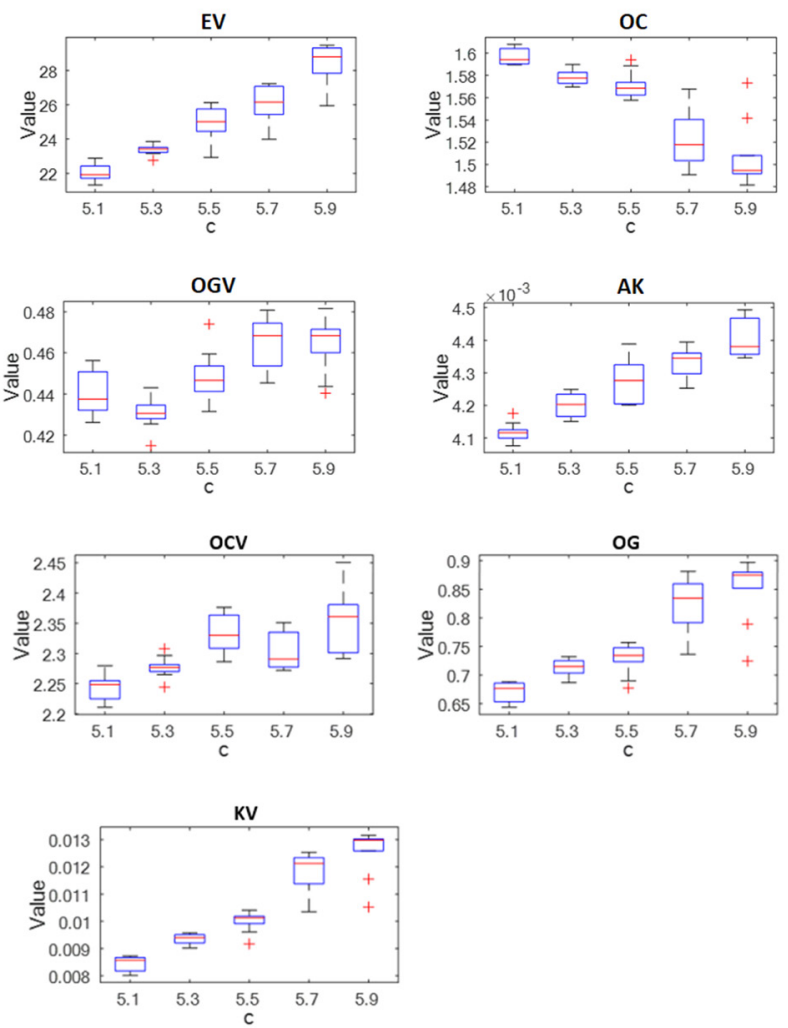

Figure 2. Boxplot of the Features for 100 Initial Conditions and Five Values of the Lorenz System. 
Lorenz system parameters $(r)$ and 100-times iterations for random initial points. The results show that the distribution of the features of each condition is significantly separable from another parameter. We applied two-sample $t$ test for each pair of parameters. Two-sample $t$ test examines the null hypothesis that the population means related to two independent, random samples from an approximately normal distribution are equal. To use an unpaired $t$ test, the population must have a normal distribution. To test the normality, the Jarque-Bera test was used. This test was applied to each distribution of the feature values for different Lorentz parameters. Jarque-Bera returns the $P$ value that is larger than 0.05 if the feature has a normal distribution. The results indicate that most features had a normal distribution, and we can apply the two-sample $t$ test.

Figure 3 shows the results of the two-sample $t$ test. The
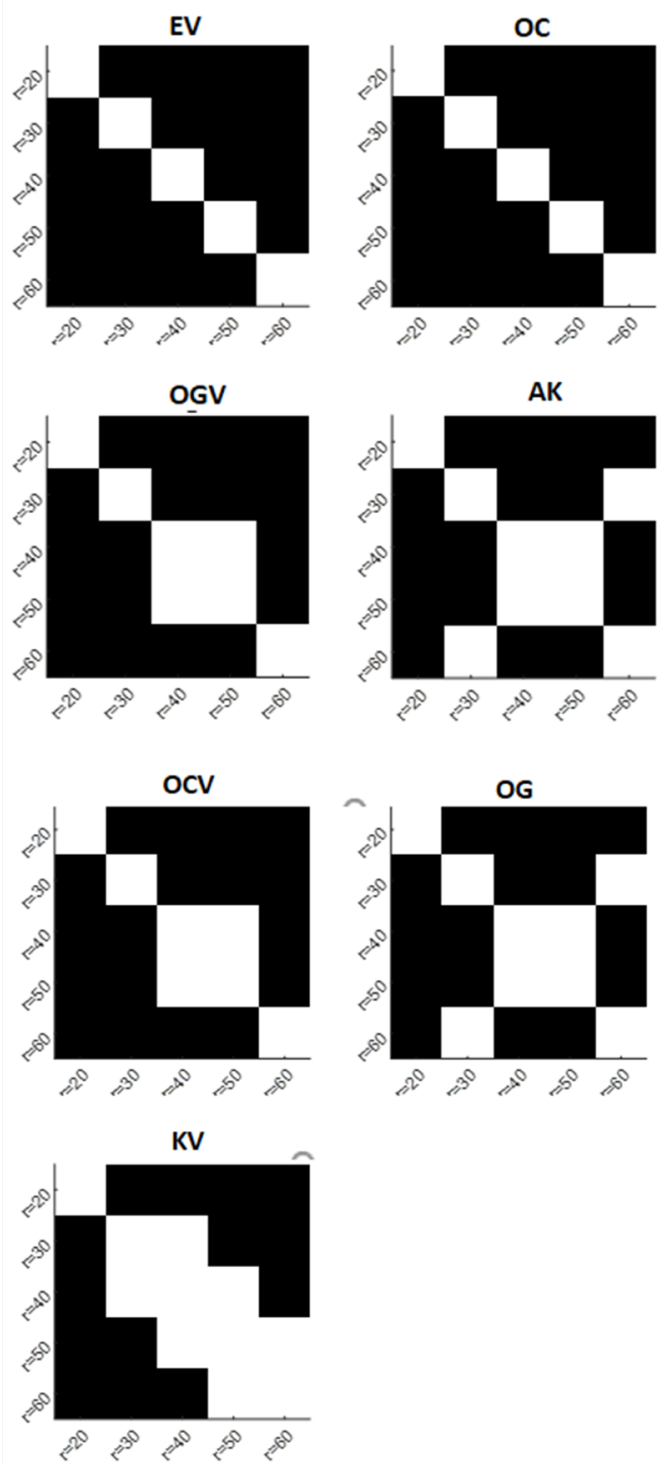

Figure 3. The $T$ Test for All Two Parameters for Geometrical Features From the Lorenz System. The black box indicates that features are independent. black box indicates that the $P$ value of the $t$ test for two pairs of the different parameter is less than 0.05 and the two distributions are significantly separable from each other. Almost all the boxes in the table for each quantifier are black and satisfy the hypothesis of two-test methods.

In the next step, the time evolution in transition from pre-seizure state to epileptic seizure was investigated. The results showed that there were significant changes in the geometrical value. For instance, the time variations of four features with better statistical results were demonstrated in Figure 4. The results indicate that the value of the geometric features increased significantly in this transition, and the onset of SWDs is easily detected by

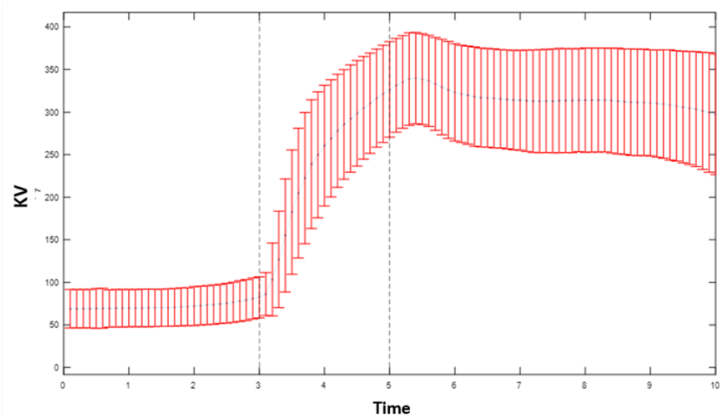

(a)

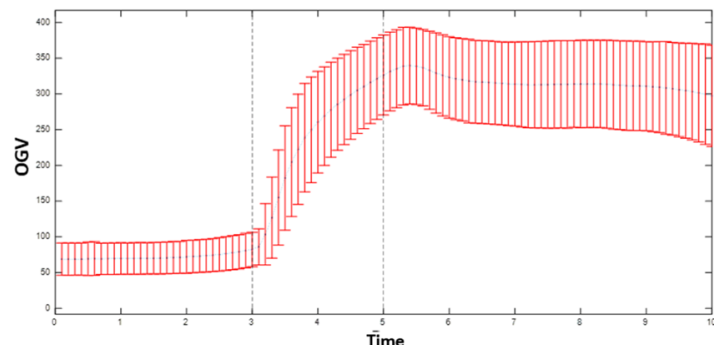

(b)
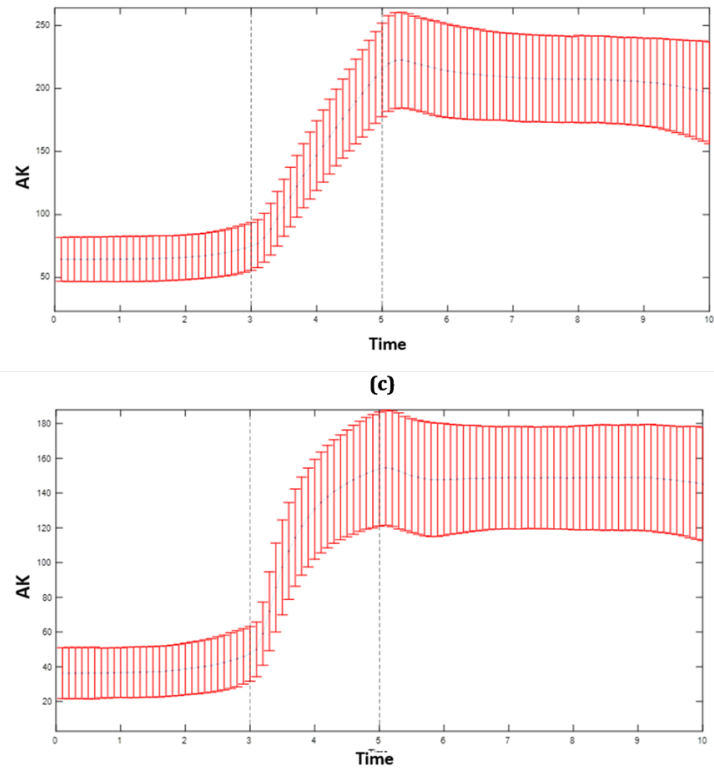

(d)

Figure 4. Time Variation of Features in Transition From a Normal State to Absence Seizure for 940 ECoG Segments. (a) KV, (b) OGV, (c) AK (d) EV. 
using these features.

OGV, (c) AK (d) EV.

In the next step, the accuracy of SWDs detection and prediction time for different threshold values was calculated. Table 1 presents the maximum accuracy for each feature and prediction time in maximum accuracy.

\section{Discussion and Conclusion}

In this study, we proposed a strong method for offline prediction of SWDs. Table 2 comprises the result of previous SWDs detection studies on animal absence epilepsy and the proposed method.

This method can play an important role in detecting and predicting epileptic seizures. To apply suitable medication methods, a doctor needs to review EEG signals, which may take hours. In addition, to avoid the side effects of antiepileptic drugs designing a close-loop system is highly desired, and the real-time stimulation can be applied when a seizure occurs. Another application of an automatic detection system is designing a warning system to protect the patient from seizures during his life. The result shows that the accuracy of the proposed method is better than the previous approaches. This study focused on the genetic epilepsy model because it is the model of

Table 1. The Average Accuracy for Each Feature in 940 ECoG Segments and the Prediction Time in Max Accuracy for Each Feature

\begin{tabular}{lccc}
\hline Feature & Symbol & ACC $(\%)$ & Prediction Time (s) \\
\hline 1 & EV & 98.23 & 1.60 \\
2 & C & 92 & 1.23 \\
3 & G & 94.3 & 1.18 \\
4 & OC & 80 & 1.32 \\
5 & OG & 97.7 & 1.54 \\
6 & OCV & 85.53 & 1.13 \\
7 & OGV & 97.23 & 1.71 \\
8 & KV & 96.17 & 1.65 \\
9 & AK & 97.32 & 1.44 \\
\hline
\end{tabular}

Table 2. The Accuracy of SWDs Detection on the Animal Model Dataset and the Proposed Method

\begin{tabular}{|c|c|c|}
\hline Authors & Year & Accuracy $(\%)$ \\
\hline Jandó et al ${ }^{39}$ & 1993 & 93 \\
\hline Van Hese et $\mathrm{al}^{4}$ & 2009 & 98 \\
\hline Xanthopoulos et al ${ }^{40}$ & 2009 & 90 \\
\hline Sitnikova et al ${ }^{10}$ & 2009 & 97.7 \\
\hline Van Hese et $\mathrm{al}^{41}$ & 2008 & 97 \\
\hline Startceva et $\mathrm{al}^{37}$ & 2015 & 94.8 \\
\hline Pablo et al $^{42}$ & 2019 & 100 \\
\hline Pedro et $\mathrm{al}^{43}$ & 2018 & 93.5 \\
\hline Lashkari et al $^{31}$ & 2018 & 97 \\
\hline The proposed method & 2021 & 98.23 \\
\hline
\end{tabular}

epilepsy and not the model of epileptic seizures such as acute chemical and electrical kindling. Because of the similar pharmacological and electrical characteristics in an animal model and human epilepsy, the results of these studies are more reliable and can be developed for clinical application.

The main focus of this study was extracting geometrical features from phase space. The effectiveness of the nonlinear approaches is demonstrated in several studies. ${ }^{37-39}$ The results support this issue. The proposed algorithm is robust to the initial point changes and significant changes with the changes in system parameters and so the features can quantify the chaotic behavior. Similar to the studies on geometrical quantifiers, the geometrical feature was significantly changed in the transition from a normal state to epileptic seizures.

This article proposed a novel offline method. However, since few samples are needed to calculate the features, we can develop the method for online application. In addition, the threshold method is simple and fast with high accuracy, and we can use this method in online mode. Another limitation of this study is the low number of subjects. The excellent performance of this proposed algorithm opens new perspectives to develop automatic techniques in clinical settings. Future studies can focus on the other genetic model of absence epilepsy like GARES. Future studies can also search for a method that can be used in online applications.

\section{Conflict of Interest Disclosures}

The authors declare that they have no conflict of interests.

\section{Ethical Statement}

This experimental study was done in the animal laboratory of Ferdowsi University, Mashhad, Iran. It was approved by the natural ethics committee (IR.NKUMS.REC.00.036). The animals were housed in pairs with free access to water and food. After surgery, they were housed individually. All procedures were done in accordance with the national guidelines for animal care and handling. Efforts were made to minimize the pain/ distress experienced by the animals. Also, the least possible number of animals necessary to produce reliable data was used.

\section{Funding}

This research was supported by grant NS400032 from the North Khorasan University of medical sciences.

\section{Acknowledgements}

The authors thank all the managers and staff of the Animal Laboratory of Ferdowsi University, Mashhad, Iran.

\section{References}

1. van Luijtelaar G, Sitnikova E, Littjohann A. On the origin and suddenness of absences in genetic absence models. Clin EEG Neurosci. 2011;42(2):83-97. doi: 


\subsection{7/155005941104200209.}

2. Kelly KM. Spike-wave discharges: absence or not, a common finding in common laboratory rats. Epilepsy Curr. 2004;4(5):176-7. doi: 10.1111/j.1535-7597.2004.04503.x.

3. Danober L, Deransart C, Depaulis A, Vergnes $M$, Marescaux C. Pathophysiological mechanisms of genetic absence epilepsy in the rat. Prog Neurobiol. 1998;55(1):2757. doi: 10.1016/s0301-0082(97)00091-9.

4. Van Hese P, Martens JP, Waterschoot L, Boon P, Lemahieu I. Automatic detection of spike and wave discharges in the EEG of genetic absence epilepsy rats from Strasbourg. IEEE Trans Biomed Eng. 2009;56(3):706-17. doi: 10.1109/ tbme.2008.2008858.

5. Luders HO, Noachtar S. Atlas of epileptic seizures and syndromes. AJNR Am J Neuroradiol. 2001;22(10):1976.

6. Buteneers P, Schrauwen B, Verstraeten D, Stroobandt D. Real-time epileptic seizure detection on intra-cranial rat data using reservoir computing. In: Köppen M, Kasabov $\mathrm{N}$, Coghill G, eds. International Conference on Neural Information Processing. Berlin, Heidelberg: Springer; 2008.

7. Casillas-Espinosa PM, Sargsyan A, Melkonian D, O’Brien TJ. A universal automated tool for reliable detection of seizures in rodent models of acquired and genetic epilepsy. Epilepsia. 2019;60(4):783-91. doi: 10.1111/epi.14691.

8. Andrade P, Paananen T, Ciszek R, Lapinlampi N, Pitkänen A. Algorithm for automatic detection of spontaneous seizures in rats with post-traumatic epilepsy. J Neurosci Methods. 2018;307:37-45. doi: 10.1016/j.jneumeth.2018.06.015.

9. Westerhuis F, Van Schaijk W, Van Luijtelaar G. Automatic detection of spike-wave discharges in the cortical EEG of rats. In: Spruijt B, Mos J, Noldus L, Sams-Dodd F, van der Staay FJ, eds. Measuring Behavior '96, International Workshop on Methods and Techniques in Behavioral Research. Wageningen: Noldus Information Technology; 1996.

10. Sitnikova E, Hramov AE, Koronovsky AA, van Luijtelaar G. Sleep spindles and spike-wave discharges in EEG: Their generic features, similarities and distinctions disclosed with Fourier transform and continuous wavelet analysis. J Neurosci Methods. 2009;180(2):304-16. doi: 10.1016/j. jneumeth.2009.04.006.

11. Fanselow EE, Reid AP, Nicolelis MA. Reduction of pentylenetetrazole-induced seizure activity in awake rats by seizure-triggered trigeminal nerve stimulation. J Neurosci. 2000;20(21):8160-8. doi: 10.1523/ jneurosci.20-21-08160.2000.

12. Wijayanto I, Hartanto R, Nugroho HA. Higuchi and Katz Fractal Dimension for Detecting Interictal and Ictal State in Electroencephalogram Signal. In: 2019 11th International Conference on Information Technology and Electrical Engineering (ICITEE). Pattaya, Thailand: IEEE; 2019. doi: 10.1109/iciteed.2019.8929940.

13. Jirka J, Prauzek M, Krejcar O, Kuca K. Automatic epilepsy detection using fractal dimensions segmentation and
GP-SVM classification. Neuropsychiatr Dis Treat. 2018;14:2439-49. doi: 10.2147/ndt.s167841.

14. Preißl H, Lutzenberger W, Pulvermüller F, Birbaumer N. Fractal dimensions of short EEG time series in humans. Neurosci Lett. 1997;225(2):77-80. doi: 10.1016/s03043940(97)00192-4.

15. Arunkumar N, Kumar KR, Venkataraman V. Entropy features for focal EEG and non focal EEG. J Comput Sci. 2018;27:440-4. doi: 10.1016/j.jocs.2018.02.002.

16. Zhao Q, Jiang H, Hu B, Li Y, Zhong N, Li M, et al. Nonlinear dynamic complexity and sources of resting-state EEG in abstinent heroin addicts. IEEE Trans Nanobioscience. 2017;16(5):349-55. doi: 10.1109/tnb.2017.2705689.

17. Silva C, Pimentel IR, Andrade A, Foreid JP, Ducla-Soares E. Correlation dimension maps of EEG from epileptic absences. Brain Topogr. 1999;11(3):201-9.

18. Eagleman SL, Vaughn DA, Drover DR, Drover CM, Cohen MS, Ouellette NT, et al. Do complexity measures of frontal EEG distinguish loss of consciousness in geriatric patients under anesthesia? Front Neurosci. 2018;12:645. doi: 10.3389/fnins.2018.00645.

19. Namazi H, Jafari S. Age-based variations of fractal structure of EEG signal in patients with epilepsy. Fractals. 2018;26(4):1850051. doi: 10.1142/s0218348x18500512.

20. Wang Q, Li Y, Liu X. Analysis of feature fatigue EEG signals based on wavelet entropy. Intern J Pattern Recognit Artif Intell. 2018;32(8):1854023. doi: 10.1142/ s021800141854023x.

21. Kang J, Chen H, Li X, Li X. EEG entropy analysis in autistic children. J Clin Neurosci. 2019;62:199-206. doi: 10.1016/j. jocn.2018.11.027.

22. Liang Z, Wang Y, Sun X, Li D, Voss LJ, Sleigh JW, et al. EEG entropy measures in anesthesia. Front Comput Neurosci. 2015;9:16. doi: 10.3389/fncom.2015.00016.

23. Srinivasan V, Eswaran C, Sriraam N. Approximate entropybased epileptic EEG detection using artificial neural networks. IEEE Trans Inf Technol Biomed. 2007;11(3):28895. doi: 10.1109/titb.2006.884369.

24. Yakovleva TV, Kutepov IE, Karas AY, Yakovlev NM, Dobriyan VV, Papkova IV, et al. EEG analysis in structural focal epilepsy using the methods of nonlinear dynamics (Lyapunov exponents, Lempel-Ziv complexity, and multiscale entropy). ScientificWorldJournal. 2020;2020:8407872. doi: 10.1155/2020/8407872.

25. Kutepov IE, Dobriyan VV, Zhigalov MV, Stepanov MF, Krysko AV, Yakovleva TV, et al. EEG analysis in patients with schizophrenia based on Lyapunov exponents. Inform Med Unlocked. 2020;18:100289. doi: 10.1016/j. imu.2020.100289.

26. Dahal P, Avagyan $M$, Skardal PS, Blaise HJ, Ning T. Characterizing chaotic behavior of REM sleep EEG using Lyapunov exponent. In: 2017 10th International Congress on Image and Signal Processing, BioMedical Engineering and Informatics (CISP-BMEI). Shanghai, China: IEEE; 2017. doi: 10.1109/cisp-bmei.2017.8302215. 
27. Hekmatmanesh A, Asl RM, Wu H, Handroos H. EEG control of a bionic hand with imagination based on chaotic approximation of largest Lyapunov exponent: a single trial BCI application study. IEEE Access. 2019;7:105041-53. doi: 10.1109/access.2019.2932180.

28. Webber C, Marwan N. Recurrence Quantification Analysis: Theory and Best Practices. Cham: Springer; 2015.

29. Webber CL Jr, Zbilut JP. Recurrence quantification analysis of nonlinear dynamical systems. Tutorials in Contemporary Nonlinear Methods for the Behavioral Sciences. 2005;94(2005):26-94.

30. Lashkari S, Sheikhani A, Hashemi Golpayegan MR, Moghimi A, Kobravi H. Topological feature extraction of nonlinear signals and trajectories and its application in EEG signals classification. Turk J Elec Eng Comp Sci. 2018;26(3):1329-42. doi: 10.3906/elk-1708-59.

31. Lashkari S, Sheikhani A, Hashemi Golpayegani MR, Moghimi A, Kobravi H. Detection and prediction of absence seizures based on nonlinear analysis of the EEG in Wag/Rij animal model. Int Clin Neurosci J. 2018;5(1):21-7. doi: 10.15171/icnj.2018.05.

32. Paxinos G, Watson C. The Rat Brain in Stereotaxic Coordinates: Hard Cover Edition. Elsevier; 2006.

33. Younessi Heravi MA, Maghooli K, Nowshiravan Rahatabad F, Rezaee R. Application of a neural interface for restoration of leg movements: intra-spinal stimulation using brain electrical activity in spinally injured rabbits. J Appl Biomed. 2020;18(2-3):33-40. doi: 10.32725/jab.2020.009.

34. Younessi Heravi MA, Pishghadam M, Raoufian $H$, Gazerani A. Recurrence quantification analysis of electrooculography signal to a control question test: a new approach for the detection of deception. Biomed Eng Appl Basis Commun. 2020;32(4):2050029. doi: 10.4015/ s1016237220500295.

35. Kennel MB, Brown R, Abarbanel HD. Determining embedding dimension for phase-space reconstruction using a geometrical construction. Phys Rev A. 1992;45(6):340311. doi: 10.1103/physreva.45.3403.

36. Fraser AM, Swinney HL. Independent coordinates for strange attractors from mutual information. Phys Rev A Gen Phys. 1986;33(2):1134-40. doi: 10.1103/physreva.33.1134.

37. Hopfengärtner R, Kasper BS, Graf W, Gollwitzer S, Kreiselmeyer G, Stefan H, et al. Automatic seizure detection in long-term scalp EEG using an adaptive thresholding technique: a validation study for clinical routine. Clin Neurophysiol. 2014;125(7):1346-52. doi: 10.1016/j. clinph.2013.12.104.

38. Rössler OE. An equation for continuous chaos. Phys Lett A. 1976;57(5):397-8. doi: 10.1016/0375-9601(76)90101-8.

39. Jandó G, Siegel RM, Horváth Z, Buzsáki G. Pattern recognition of the electroencephalogram by artificial neural networks. Electroencephalogr Clin Neurophysiol. 1993;86(2):100-9. doi: 10.1016/0013-4694(93)90082-7.

40. Xanthopoulos P, Liu CC, Zhang J, Miller ER, Nair SP, Uthman BM, et al. A robust spike and wave algorithm for detecting seizures in a genetic absence seizure model. In: 2009 Annual International Conference of the IEEE Engineering in Medicine and Biology Society. Minneapolis, MN, USA: IEEE; 2009. doi: 10.1109/iembs.2009.5334941.

41. Van Hese P, Vanrumste B, Hallez H, Carroll GJ, Vonck K, Jones RD, Bones PJ, D’Asseler Y, Lemahieu I. Detection of focal epileptiform events in the EEG by spatio-temporal dipole clustering. Clin Neurophysiol 2008; 119:1756-1770.

42. Pablo M.C, Sargsyan, A., Melkonian, D., \& O’Brien, T. J. A universal automated tool for reliable detection of seizures in rodent models of acquired and genetic epilepsy. Epilepsia. 2019; 60(4): 783-791.

43. Pedro A, Tomi P, Robert C, Niina L, Asla P. Algorithm for automatic detection of spontaneous seizures in rats with post-traumatic epilepsy, J Neurosci Methods. 2018; Sep 1: 30, 37-45. doi: 10.1016/j.jneumeth.2018.06.015. 